Ann. Sci. forest., 1970, 27 (3), 231-242.

\title{
RELATIONS PHÉNOTYPIQUES AU STADE JUVÉNILE ENTRE LA DENSITÉ DU BOIS ET DIVERS CARACTÈRES PHÉNOLOGIQUES ET DE VIGUEUR CHEZ PICEA ABIES KARST.
}

\author{
J.-F. LACAZE et H. POLGE \\ Station d'Amélioration des Arbres forestiers, \\ Station de Recherches sur la Qualité des Bois, \\ Centre national de Recherches forestières, 54-Nancy \\ Institut national de la Recherche agronomique
}

\section{RESUME}

Sur des semis d'épicéas de 4 ans de 16 provenances différentes, la densité du bois est corrélée de façon négative, au niveau des populations, avec le diamètre et la hauteur (liaisons non significatives) ainsi qu'avec la tardiveté du débourrement (liaisons significatives). La corrélation avec la fréquence des pousses d'août est positive mais non significative. Des liaisons analogues existent au niveau individuel à l'intérieur des populations.

\section{INTRODUCTION}

Pour améliorer l'efficacité des opérations de sélection de populations ou d'individus, il convient généralement de ne choisir - au moins dans un premier stade qu'un nombre limité de critères. Ainsi, en France, les programmes d'amélioration concernant l'épicéa de basse ou moyenne altitude ne retiennent que deux caractères: vigueur et débourrement végétatif tardif.

En l'absence de toute connaissance sur les liaisons génétiques existant entre les critères choisis et d'autres caractères non retenus à ce premier stade mais présentant une certaine importance, la sélection comporte un risque : celui d'effectuer une sélection indirecte contre ces autres caractères.

Nous avons rendu compte précédemment des résultats d'une expérimentation sur les provenances d'épicéa dont le bilan a été tiré au stade pépinière (quatre ans) (LACAzE, 1969), en mettant l'accent sur les caractères vigueur et débourrement végétatif au niveau des populations et au niveau individuel. Notre propos a pour objectif d'explorer les relations existant entre ces critères et divers caractères phénologiques (durée de l'élon- 
gation, pousse d'août) d'une part, et d'autre part un caractère technologique classique, la densité du bois ; celui-ci n'a pas été retenu comme critère principal, mais son importance économique est telle qu'il serait particulièrement fâcheux qu'un programme d'amélioration aboutisse indirectement, pour ce qui le concerne, à une sélection négative. Les relations étudiées sont phénotypiques, ce qui signifie qu'elles pourraient s'écarter, malgré la faible variabilité du milieu en pépinière, des corrélations génétiques additives. Nous ignorons en effet la part de la corrélation due aux intéractions génétiques. Les résultats fournis n'indiqueront donc que des tendances.

\section{MATERIEL ET METHODE}

L'analyse a porté sur 16 provenances, en majorité de basse et moyenne altitude, dont on trouvera les caractéristiques sur le tableau 1.

Il s'agissait d'un dispositif en blocs complets à 5 répétitions, les mesures étant effectuées sur échantillon de 10 plants par bloc, soit 50 plants par provenance.

Nature des mesures et observations:

- vigueur: hauteur et diamètre à 4 ans,

- phénologiques: pousse d'août et débourrement végétatif (au cours de la quatrième année),

- densité.

Le matériel utilisé pour les déterminations de densité a consisté en fragments de tiges prélevés à environ $5 \mathrm{~mm}$ au-dessus du collet, afin d'éviter l'influence des anomalies de structure qui se rencontrent à ce niveau; la longueur des échantillons (voisine de $3 \mathrm{~cm}$ ) avait été choisie de façon à permettre une précision relative des mesures satisfaisante, sans cependant atteindre le niveau des premières branches importantes où l'accumulation de nœuds vient fausser la densité du bois de tige.

Les trois derniers accroissements annuels (1963 à 1965) étaient présents dans la quasitotalité des échantillons.

Malgré ces précautions, la mesure des densités sur des fragments de tiges, dont certains n'atteignaient pas $3 \mathrm{~mm}$ de diamètre, a soulevé de nombreux problèmes :

La méthode dite «de la saturation intégrale » était délicate à employer : elle consiste, rappelons-le, à remplir d'eau toutes les cavités cellulaires (soit par le vide, soit par ébullition) et à calculer la densité à l'aide d'une formule appropriée à partir du poids maximal ainsi obtenu et du poids anhydre. Or, lorsque la dimension axiale des échantillons représente plusieurs longueurs de fibres, la saturation se fait mal, et l'on peut aboutir à des valeurs de densité trop élevées.

Une autre méthode, reposant sur la détermination directe du volume saturé au voluménomètre à mercure, a été adoptée comme méthode de base, mais son application a soulevé également certaines difficultés du fait de la faible taille des échantillons: la précision obtenue est relativement faible $\left(3 \mathrm{~mm}^{3}\right)$ et, de plus, des bulles d'air, susceptibles de fausser les résultats, peuvent se produire lorsque le mercure utilisé d'une part, ou le tube capillaire d'autre part, ne sont pas parfaitement propres.

Pour cette raison, il a été procédé, sur chacun des 800 échantillons, à un minimum de quatre déterminations successives de la densité :

- la première (D1) par la méthode de saturation au vide pendant 4 jours,

- la deuxième (D2) par la méthode au voluménomètre à mercure,

- les troisième et quatrième (D3 et D4) par la variante «ébullition» de la méthode de saturation pendant 48 heures.

Pour la première, le vide a été préféré à l'ébullition, car il n'entraîne ni solubilisation importante, ni modification de forme de l'échantillon susceptible d'affecter par la suite les mesures de volume. Par ailleurs, il paraissait préférable de commencer par une méthode de saturation car il arrive, qu'après les mesures au voluménomètre, les échantillons conservent quelques traces de mercure qui, du fait de son poids spécifique élevé, peuvent fausser considérablement les pesées ultérieures. 
TABLEAU 1

Description des provenances

Provenances description

\begin{tabular}{|c|c|c|c|c|c|c|c|c|c|}
\hline Nom & $\begin{array}{l}\text { Abrévia- } \\
\text { tion }\end{array}$ & Région & $\begin{array}{l}\text { Longitude } \\
\text { Est }\end{array}$ & $\begin{array}{l}\text { Latitude } \\
\text { Nord }\end{array}$ & $\begin{array}{l}\text { Altitude } \\
(\mathrm{m})\end{array}$ & $\begin{array}{c}\text { Pluviosité } \\
\text { de la } \\
\text { station } \\
\text { la plus } \\
\text { proche } \\
\text { (mm) }\end{array}$ & Roche-mère & $\begin{array}{r}\mathrm{N} \\
\mathrm{ou} \\
\mathrm{A}\end{array}$ & $\begin{array}{c}\mathrm{N}^{0} \text { du peuple- } \\
\text { ment porte- } \\
\text { graines }\end{array}$ \\
\hline Bonnétage II .... & BONE II & Jura. $2^{e}$ p'ateau & $6^{\circ} 45$, & $47^{\circ} 10^{\prime}$ & 875 & 1.390 & Calcaire & $\mathrm{N}$ & $25-29$ \\
\hline Les Frasses $\ldots \ldots$ & FRAS & Préalpes du Norc & $6^{\circ} 36^{\prime}$ & $45^{\circ} 52^{\prime}$ & 1.500 & $\left(\begin{array}{lll}\text { (a } & 1304\end{array}\right)$ & Schistes & iN & $74-34$ \\
\hline Morzine $\ldots \ldots \ldots$ & MORZ & $\begin{array}{l}\text { Préalpes du Nord } \\
\text { Haute altitude }\end{array}$ & $6^{\circ} 48^{\prime}$ & $46^{\circ} 12^{\prime}$ & $1.700 / 1.800$ & & Flysch & IN & $74-30$ \\
\hline Plan Bois ........ & PL-BO & $\begin{array}{l}\text { Préalpes du Nord } \\
\text { Basse altitude }\end{array}$ & $6^{\circ} 27^{\prime}$ & $46^{\circ} 19^{\prime}$ & 500 & 930 & Argilo-calcaire & N & \\
\hline Gérardmer I & GERA I & $\begin{array}{l}\text { Vosges. Chaîne } \\
\text { centrale }\end{array}$ & $6^{\circ} 54^{\prime}$ & $48^{\circ} 11^{\prime}$ & $650 / 750$ & 1.530 & Granite & $N$ & \\
\hline Gorowo-Ilawieckiz & GO-IL & $\begin{array}{l}\text { Po!ogne. } \\
\text { Nord-Est }\end{array}$ & $20^{\circ} 30^{\prime}$ & $54^{\circ} 17^{\prime}$ & 150 & 660 & & $\mathrm{~N}$ & \\
\hline Sobowidze $\ldots \ldots$ & 5OBO & Pologne. Nord & $18^{\circ} 30^{\prime}$ & $54^{\circ} 10^{\prime}$ & 120 & 575 & Sables & A & \\
\hline Borki $\ldots$ & BORK & $\begin{array}{l}\text { Pologne. Nord- } \\
\text { Est. }\end{array}$ & $22^{\circ} 03^{\prime}$ & $54^{\circ} 06^{\prime}$ & 160 & 618 & $\begin{array}{l}\text { argileux } \\
\text { Argilo- }\end{array}$ & $\mathrm{N}$ & \\
\hline Plaska ... & PLAS & $\begin{array}{l}\text { Pologne. Nord- } \\
\text { Est. }\end{array}$ & $23^{\circ} 13^{\prime}$ & $53^{\circ} 54^{\prime}$ & 120 & 586 & $\begin{array}{l}\text { Sables faible } \\
\text { ment argileux. }\end{array}$ & $\mathrm{N}$ & \\
\hline Zwierzyniec . & ZWIE & $\begin{array}{l}\text { Pologne. Nord- } \\
\text { Est. }\end{array}$ & $23^{\circ} 45^{\prime}$ & $52^{\circ} 42^{\prime}$ & 150 & 585 & $\begin{array}{l}\text { Sables } \\
\text { argileux }\end{array}$ & $\mathrm{N}$ & \\
\hline Bialowieza $\ldots \ldots$ & BIAL & $\begin{array}{l}\text { Pologne. Nord } \\
\text { Est. }\end{array}$ & $23^{\circ} 52^{\prime}$ & $52^{\circ} 42^{\prime}$ & 150 & 585 & $\begin{array}{l}\text { Sables } \\
\text { argileux }\end{array}$ & $N$ & \\
\hline Tarnawa $\quad \ldots \ldots \ldots$ & TARN & $\begin{array}{l}\text { Pologne. Carpa- } \\
\text { thes de l'Est }\end{array}$ & $22^{\circ} 50^{\prime}$ & $49^{\circ} 05^{\prime}$ & 750 & 1.035 & & $\mathrm{~N}$ & \\
\hline Istebna II $\ldots \ldots \ldots$ & ISTE II & $\begin{array}{l}\text { Pologne. Beskide: } \\
\text { Slaski }\end{array}$ & $18^{\circ} 50^{\prime}$ & $49^{\circ} 35^{\prime}$ & 660 & 1.050 & & $N$ & \\
\hline Orawa $\ldots \ldots \ldots$ & ORAW & $\begin{array}{l}\text { Pologne. Beskides } \\
\text { Slaski }\end{array}$ & $19^{\circ} 35^{\prime}$ & $49^{\circ} 35^{\prime}$ & 950 & & & $N$ & \\
\hline $\begin{array}{l}\text { Wilna-Minsk } \ldots \ldots \\
\text { Brdy } \ldots \ldots\end{array}$ & $\begin{array}{l}\text { WL-MN } \\
\text { BRDY }\end{array}$ & $\begin{array}{l}\text { U.R.S.S. } \\
\text { Tchécoslovaquie } \\
\text { Bohême }\end{array}$ & $13^{\circ} 50^{\prime}$ & $49^{\circ} 40^{\circ}$ & 800 & & & $\mathrm{~N}$ & $\begin{array}{l}\text { (Peuple- } \\
\text { mant relique) }\end{array}$ \\
\hline
\end{tabular}


Pour les raisons indiquées plus haut (difficulté d'obtenir une saturation intégrale sur des échantillons de grande longueur), D1 devait, sauf erreurs de manipulation, être au moins égal à D2.

Il en était normalement de même pour D3 et D4 qui, en outre, devaient théoriquement aller en diminuant par rapport à D1 par suite de la solubilisation dans l'eau bouillante d'un certain nombre de substances chimiques imprégnant les membranes cellulaires.

De nouvelles mesures de D2: D’2, ont été faites :

- soit lorsque D1, D3 et D4 étaient concordants entre eux, mais différents de D2, quel que soit le sens de variation,

- soit lorsqu'une seule des valeurs de D1, D3 ou D4 était inférieure à D2.

La densité finalement retenue a toujours été, soit $\mathrm{D} 2$, soit $\mathrm{D}^{\prime} 2$, lorsque les vérifications ainsi faites (qui intéressaient environ $10 \%$ des échantillons) avaient montré que la première mesure au voluménomètre avait été erronée. Dans l'un et l'autre cas, il s'agissait de l'infradensité : rapport du poids anhydre au volume saturé.

Les mesures des diamètres ont été faites à l'aide d'un pied à coulisse au milieu des échantillons ayant servi aux déterminations de densité, c'est-à-dire à environ $2 \mathrm{~cm}$ au-dessus du collet ; deux diamètres perpendiculaires ont été pris si nécessaire, et la précision obtenue était du $1 / 10^{\circ}$ de millimètre.

\section{RESULTATS}

\section{1. - Classement pour la densité du bois}

Celui-ci est résumé ci-dessous.

\begin{tabular}{ll|l|l|} 
MORZ & 573,8 \\
FRAS & 564,5 \\
BRDY & 553,6 \\
PL-BO & 549,9 \\
GERA & 546,1 \\
BONE & 542,0 \\
SOBO & 539,6 & \\
ORAW & 538,5 & \\
BORK & 529,2 & \\
ISTE, GO-IL & 527,8 & \\
PLAS & 526,8 & \\
WL-MN & 521,8 & \\
ZWIE & 519,8 & \\
BIAL & 518,6 & \\
TARN & 505,0 & \\
\end{tabular}

On remarque en tête du classement toutes les provenances françaises ainsi aue la provenance du domaine hercynien carpathique: Brdy.

Il est à noter que, dans ce lot de tête, figurent aussi bien des populations de haute altitude (Morzine et Les Frasses) que de moyenne et basse altitude. A l'opposé, les provenances de basse altitude du nord-est de la Pologne (domaine baltique) se situent nettement en queue du classement. 


\section{2. - Liaisons entre densité du bois et les autres caractères,} au niveau des populations

La provenance Morzine qui s'écarte trop du reste de l'échantillon de provenances, pour tous les caractères envisagés, a été éliminée de cette étude. Il convient en effet que les distributions marginales des valeurs moyennes utilisées pour les calculs de régression ne s'éloignent pas exagérément de la loi normale.

La provenance Les Frasses a également été éliminée pour la même raison dans les calculs de liaison faisant intervenir la hauteur.

\section{Densité du bois et hauteur à 4 ans.}

Le coefficient de corrélation $r=-0,21$ n'est pas significativement différent de 0 . La figure 1 permet d'interpréter ce résultat. Les provenances du groupe baltique et celles du groupe français semblent obéir à des lois indépendantes, mais l'effectif de provenances à l'intérieur de ces deux groupes est insuffisant pour tenter de les découvrir.

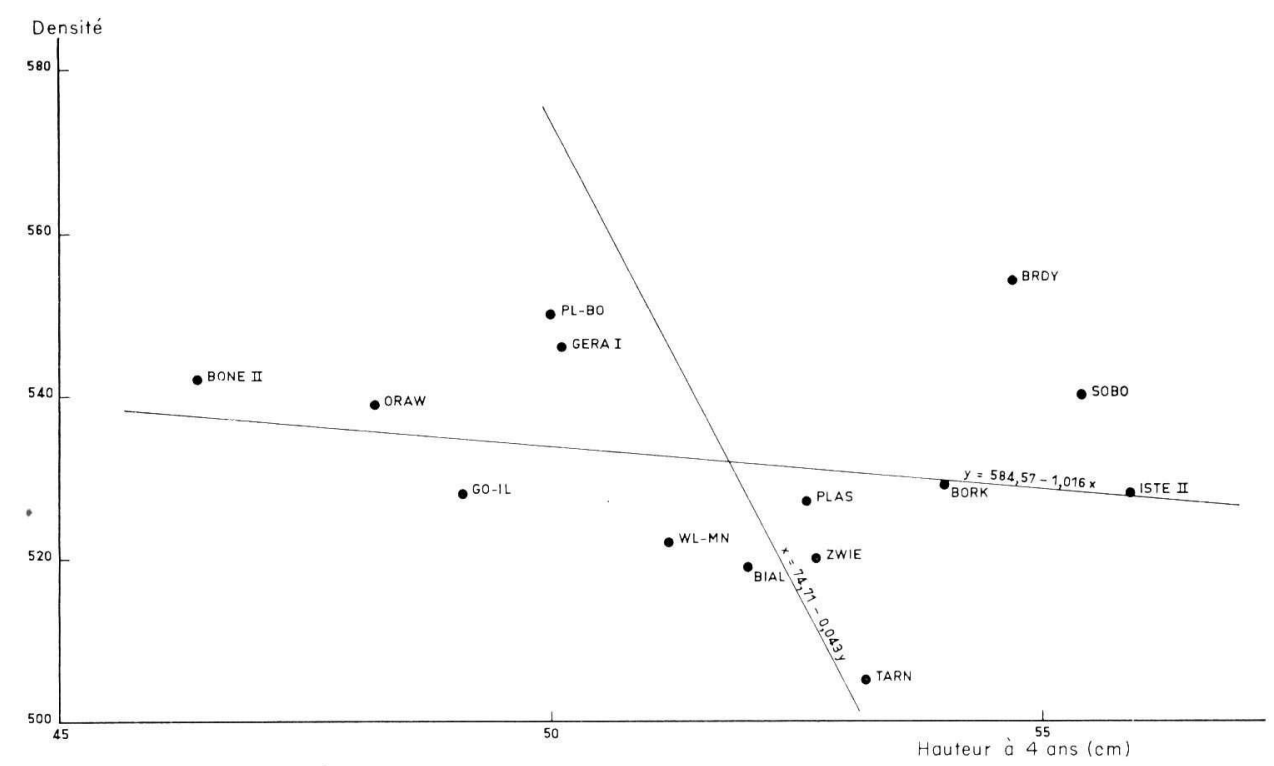

FIG. 1. - Relation entre la densité du bois et la hauteur à 4 ans (niveau provenances) FIG. 1. - Relation between wood density and 4 years height (provenances level)

On discerne ici la difficulté rencontrée pour établir une loi de variabilité infraspécifique en partant d'un échantillon réduit de populations prélevées dans les régions de provenances différentes.

Les résultats obtenus ne permettent pas de conclure à une indépendance des deux caractères; nous constatons seulement que les populations vigoureuses de l'aire française produisent un bois plus dense que celles, également vigoureuses, du domaine baltique. 


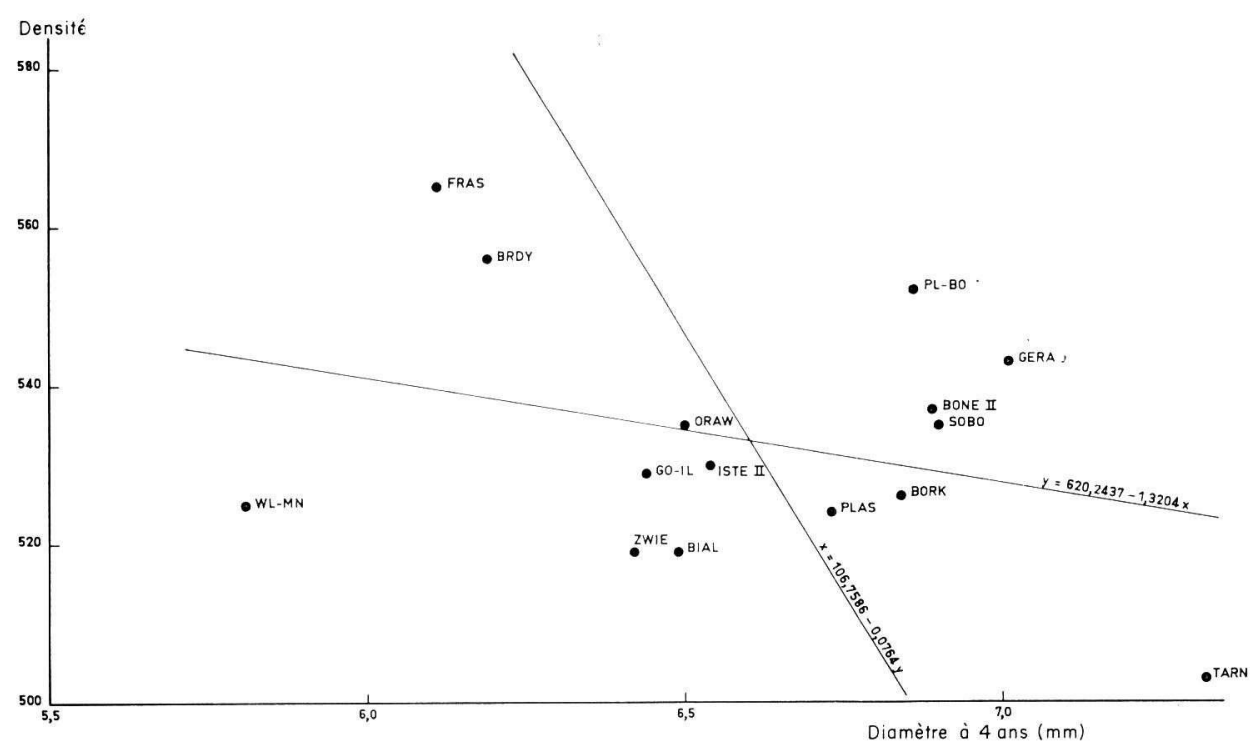

FIG. 2. - Relation entre la densité du bois et le diamètre au collet à 4 ans (niveau provenances)

FIG. 2. - Relation between wood density and collar diameter of 4 years seedlings (provenances level)

\section{Densité du bois et diamètre à 4 ans.}

Le coefficient de corrélation $r=-0,32$ n'est pas significatif; il est cependant supérieur à celui trouvé pour les liaisons entre densité du bois et hauteur à quatre ans et de même signe. L'examen de la figure 2 conduit aux mêmes conclusions que précédemment.

\section{Densité du bois et tardiveté du débourrement végétatif.}

Nous obtenons ici un coefficient de corrélation $r=-0,68^{* * *}$ (figure 3) très significatif, ce qui tendrait à prouver que les populations précoces produisent un bois plus dense. En fait, ce résultat pourrait ne pas avoir une portée générale parce que contingent à l'échantillon de provenances étudiées. Toutefois, il apparaît assez clairement que les provenances du groupe baltique sont tardives et peu denses et celles du groupe français denses et plus précoces.

Densité du bois et fréquence de pousses d'août.

On retrouve ici un coefficient de corrélation non significatif, $r=+0,39$ (figure 4), mais néanmoins assez élevé entre densité du bois et fréquence de plants avec pousse d'août. Ceci exprime une tendance, pour les populations ayant un nombre important de sujets effectuant une pousse d'août, à produire du bois plus dense.

On peut penser que le premier arrêt de la croissance en hauteur entraîne la formation d'une première zone plus ou moins large de bois dense ; puis vient la pousse d'août qui se traduit, au niveau de l'assise génératrice, par un nouvel apport d'auxines, 


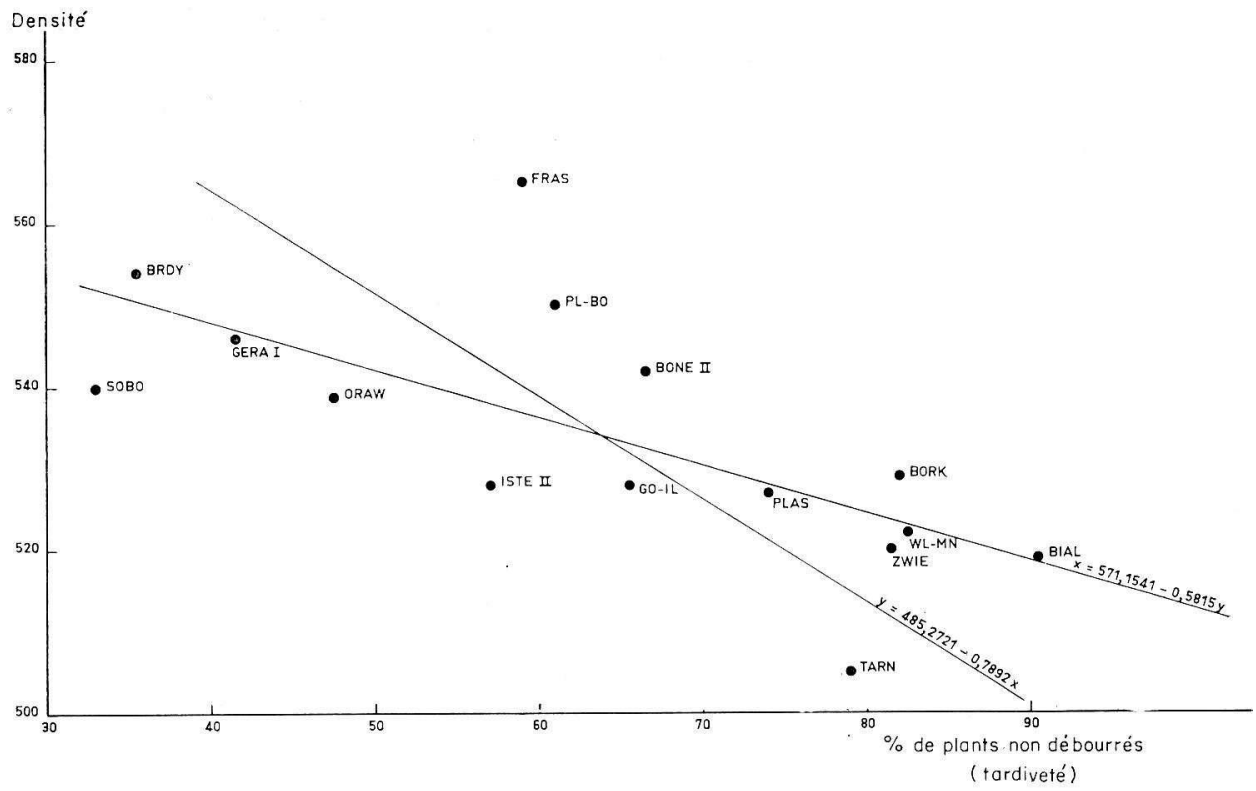

FIG. 3. - Relation entre la densité du bois et la tardiveté du débourrement végétatif (niveau provenances)

FIG. 3. - Relation between wood density and flushing rank (provenances level)



FIG. 4. - Relation entre la densité du bois et la fréquence de pousses d'août (niveau provenances) Fig. 4. - Relation between wood density and lamma shoots frequence (provenances level) 
donc par la production de trachéides à cavités cellulaires plus grandes et à membranes plus minces. Cependant, ne serait-ce qu'en raison de la durée du jour déjà réduite à cette époque de l'année, la densité du bois alors formé ne s'abaisse pas, et de très loin, jusqu'au niveau de celle du bois initial. Enfin, après l'arrêt de la pousse d'août, le bois final se développe normalement; au total, la durée de formation et la largeur du bois ayant une densité supérieure à celle du bois initial pourraient ainsi être plus élevées dans les individus ayant fait une pousse d'août.

Cette hypothèse est d'ailleurs en accord avec les observations de nombreux physiologistes; en particulier LARson (1960) a montré que, placés en jours longs, des semis de Pinus resinosa pouvaient prolonger leur période de formation du bois initial, et que des cellules analogues à celles du bois final pouvaient alors être produites artificiellement, soit par passage en jours courts, soit par une décapitation du bourgeon terminal qui supprime la production hormonale.

$\mathrm{Si}$ des semis placés en jours courts sont à nouveau transférés en jours longs, ils ne peuvent reformer des cellules de bois initial que si leur bourgeon terminal est intact, ou, dans le cas contraire, si une application d'acide indol-acétique leur est faite.

L'influence des auxines issues du bourgeon terminal sur le passage du bois initial au bois final semble ainsi prouvée; quant à leur mode d'action, il a été suggéré (LARson, 1963) que les auxines pouvaient jouer le rôle d'un plastifiant de la membrane primaire ; abondantes, elles permettraient à la cellule d'atteindre, toutes choses égales par ailleurs, des dimensions plus importantes, d'où résulteraient, pour une même quantité d'hydrates de carbone disponibles pour l'édification de la membrane secondaire, à la fois des parois cellulaires plus minces et des lumens plus larges, d'où une densité du bois moins élevée.

On peut noter encore, au sujet des croissances de type multinodal, que dans des études sur des provenances d'Abies grandis (Polge, 1968), une liaison positive très forte avait été observée entre la largeur du bois final et le pourcentage de plants ayant fait une pousse d'août $(r=+0,57$ avec 24 D.L. après transformation en arcs sinus $\checkmark$ pourcentage). Malheureusement, dans ce cas particulier, la corrélation entre largeur du bois final et largeur totale était encore plus étroite $(r=+0,92)$, en sorte qu'il n'y avait pas de liaison significative entre la densité du bois et le pourcentage de plants ayant fait une pousse d'août.

\section{Densité du bois et durée de la période d'élongation.}

Le coefficient de corrélation $r=+0,29$ n'est pas significatif. En principe, on pourrait s'attendre à obtenir un coefficient de corrélation négatif en se basant sur l'hypothèse qu'à une période d'élongation de faible durée devrait correspondre une production de bois initial moindre, donc à une densité globale supérieure. En fait, nous avons déjà noté (LACAzE, 1969) que les provenances baltiques représentées dans cette expérience se différenciaient des populations françaises, de vigueur à peu près équivalente, par une durée d'élongation notablement inférieure et par conséquent par une vitesse d'élongation supérieure.

On peut donc penser, en s'en rapportant à la théorie hormonale, qu'à une vitesse d'élongation plus rapide correspondrait un apport d'auxines, par unité de temps de fonctionnement de l'assise cambiale, plus important, donc des cellules plus grandes à 
parois plus minces, et finalement une densité propre du bois initial plus faible susceptible d'entraîner, par voie de conséquences, une baisse de la densité moyenne.

Une étude précédente (LACAZE, 1969) avait mis en évidence l'intérêt des provenances du domaine baltique (vigueur et tardiveté du débourrement). Le gain à attendre de cette sélection de provenances serait partiellement réduit par une certaine perte sur la densité du bois.

\section{3. - Liaisons entre densité du bois et divers caractères au niveau individuel}

Nous examinons maintenant les différents couples de caractères déjà envisagés, au niveau individuel, dans chaque population.

TABLEAU 2

Coefficients de corrélation Densité - Diamètre

TABLE 2

Correlation Coefficients Density - Diameter

\begin{tabular}{|c|c|}
\hline Provenances & $\begin{array}{l}\text { Coefficients } \\
\text { de corrélation }\end{array}$ \\
\hline ORAW & $-0,70$ \\
\hline WL-MN $\ldots \ldots \ldots$ & $-0,69$ \\
\hline BORK $\ldots \ldots \ldots$ & $-0,69$ \\
\hline FRAS $\ldots \ldots \ldots \ldots$ & $-0,68$ \\
\hline BIAL $\ldots \ldots \ldots \ldots$ & $-0,67$ \\
\hline SOBO $\ldots \ldots \ldots$ & $-0,65$ \\
\hline GO-1L $\ldots \ldots \ldots$ & $-0,64$ \\
\hline PLAS .......... & $-0,62$ \\
\hline MORZ .......... & $-0,61$ \\
\hline ZWIE & $-0,61$ \\
\hline GERA I $\ldots \ldots \ldots$ & $-0,60$ \\
\hline TARN $\ldots \ldots \ldots$ & $-0,57$ \\
\hline BONE II $\ldots \ldots \ldots$ & $-0,57$ \\
\hline ISTE II $\ldots \ldots \ldots$ & $-0,51$ \\
\hline PL-BO $\ldots \ldots \ldots$ & $-0,48$ \\
\hline BRDY $\ldots \ldots \ldots$ & $-0,34$ \\
\hline
\end{tabular}

Le tableau 2 fournit les coefficients de corrélation intrapopulation entre den.té du bois et diamètre. On note que ces deux caractères se lient négativement pour toutes les populations étudiées (liaison un peu moins étroite au sein de la provenance Brdy). Il est donc probable que, chez l'épicéa, une sélection individuelle au seul profit de la vigueur se traduirait par une réduction de densité du bois, ce qui ne doit pas surprendre mais constitue un handicap sérieux.

Pour étudier les liaisons entre densité du bois et débourrement (caractère qualitatif noté ici avec une échelle binaire), nous avons comparé les moyennes de densité des 
deux sous-populations tardives (non débourrées) et précoces (débourrées), le 27 avril 1966, de quelques provenances représentatives des régions de provenances étudiées.

\begin{tabular}{|c|c|c|c|}
\hline & \multicolumn{2}{|c|}{ Moyennes de densité des } & \multirow{2}{*}{ Test $\mathrm{t}$} \\
\hline & $\begin{array}{l}\text { sous-populations } \\
\text { précoces }\end{array}$ & $\begin{array}{l}\text { sous-populations } \\
\text { tardives }\end{array}$ & \\
\hline Gérardmer & 548,3 & 528,5 & $1,30 \mathrm{NS}$ \\
\hline Plan Bois $\ldots \ldots \ldots \ldots \ldots$ & 547,4 & 553,5 & $0,53 \mathrm{NS}$ \\
\hline Bonnétage $\ldots \ldots \ldots \ldots \ldots$ & 542,3 & 534,0 & $0,75 \mathrm{NS}$ \\
\hline Brdy $\ldots \ldots \ldots \ldots \ldots \ldots$ & 559,1 & 550,2 & $0,85 \mathrm{NS}$ \\
\hline Istebna $\ldots \ldots \ldots \ldots \ldots$ & 534,8 & 524,2 & $0,95 \mathrm{NS}$ \\
\hline Bialowieza $\ldots \ldots \ldots \ldots$ & 529,8 & 517,4 & $0,77 \mathrm{NS}$ \\
\hline
\end{tabular}

Les différences ne sont pas significatives, mais il apparaît une tendance : les souspopulations de plants précoces produisent, à une exception près, du bois plus dense, ce qui tend à confirmer la relation trouvée précédemment au niveau des populations. Si ce résultat était vérifié (corrélation génétique), les opérations de sélection individuelle pour la tardiveté risqueraient de conduire à une sélection négative pour la densité du bois.

Une analyse identique a été effectuée pour étudier la relation entre densité et pousse d'août :

\begin{tabular}{|c|c|c|}
\hline & \multicolumn{2}{|c|}{ Moyenne de densité des } \\
\hline & $\begin{array}{l}\text { sous-populations } \\
\text { sans pousse d'août }\end{array}$ & $\begin{array}{l}\text { sous-populations } \\
\text { avec pousse d'août }\end{array}$ \\
\hline Gérardmer $\ldots \ldots \ldots \ldots \ldots \ldots \ldots \ldots$ & 551 & 505 \\
\hline Plan Bois $\ldots \ldots \ldots \ldots \ldots \ldots \ldots$ & 549 & 557 \\
\hline Bonnétage $\ldots \ldots \ldots \ldots \ldots \ldots \ldots \ldots$ & 535 & 551 \\
\hline Brdy $\ldots \ldots \ldots \ldots \ldots \ldots \ldots \ldots$ & 554 & 557 \\
\hline 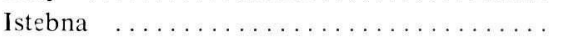 & 529 & 529 \\
\hline Bialowieza $\ldots \ldots \ldots \ldots \ldots \ldots \ldots \ldots$ & 517 & 555 \\
\hline
\end{tabular}

Les différences entre sous-populations ne sont pas significatives. Cependant, à l'exception de Gérardmer et Istebna, il semble que les plants avec pousse d'août soient légèrement plus denses que ceux qui n'en portent pas, ce qui va dans le sens des conclusions tirées au chapitre précédent.

\section{CONCLUSION}

Ces quelques résultats concernent un matériel d'étude très jeune, d'où la nécessité de les examiner avec une certaine prudence. Il semble néanmoins que - au moins chez l'épicéa - les risques de sélection indirecte, défavorables pour des caractères non retenus comme critères principaux de sélection, ne soient pas négligeables. 


\section{SUMMARY}

PHENOTYPIC RELATIONSHIP BETWEEN WOOD DENSITY AND GROWTH CRITERIA, LATE FLUSHING, LAMMA SHOOTS FROM YOUNG SPRUCE SEEDLINGS

\section{(« PICEA ABIES $\gg$ KARST)}

In France, the main selection criteria, chosen for low altitude spruce (Picea abies) breeding programs are growth and late flushing. This list is limited in view to improve the efficiency of this first stage of selection.

Nevertherless, it is important to know if the expected improvement of these two characters will not involve an indesirable indirect selection for some others traits which are not taken in account but which may have economical importance, as for instance wood density.

This experiment concerns 16 spruce provenances, mainly from low and middle altitude. The analysis has been done at two levels: between and within populations, on very young material (4 years seedlings).

Wood density was measured on $3 \mathrm{~cm}$ long stem fragments, extracted just above the collar level. Because of the very small sizes of the specimens, four determinations were made on each sample, according to the following methods:

- maximum moisture content method by using the vacuum during 4 days (measurement $\mathrm{D}_{1}$ ),

- mercury volumeter method (measurement $\mathrm{D}_{2}$ ),

- maximum moisture content method by boiling during 48 hours (measurements $D_{3}$ and $\mathrm{D}_{4}$ ).

The density data used for the analysis were $\mathrm{D}_{2}$ or a new value $\mathrm{D}_{2}$ obtained by the same method applied again, either when $D_{1}, D_{3}$ and $D_{4}$ were similar but all higher or lower than $D_{2}$, either when only one of these values was lower than $D_{2}$.

The spruce populations under study are significantly different for wood density; the baltic area provenances are less dense.

The relations obtained from population means may be summarized as follows :

Wood density is negatively correlated with size characters : the non-significant correlation coefficients are -0.21 for height (fig. 1) and -0.32 for diameter (fig. 2).

Wood density is also negatively correlated with the rank of flushing and the correlation coefficient -0.68 is significant (fig. 3 ). fig. 4).

Correlation between wood density and frequency of lamma shoots is positive $(r=+0.39$,

The validity of those regression laws for the whole species is doubtful, the number of studied provenances being too low.

At the individual level, inside populations, we found the same relations as above between wood density and other characters: growth, flushing rank and lamma shoots occurence.

We must outline that individual or population selection for the two characters chosen for low and middle altitude spruce may lead to a reduction of wood density if adequate precautions are not taken.

\section{ZUSAMMENFASSUNG}

PHANOTYPISCHE BEZIEHUNGEN IM JUGENDSTADIUM ZWISCHEN DER ROHDICHTE DES HOLZES UND VERSCHIEDENEN PHÄNOLOGISCHEN MERKMALEN SOWIE DER « WUCHSKRAFT 》 BEI DER FICHTE

(《 PICEA ABIES» KARST)

Die «Wuchskraft» (vigueur) und das Spättreiben sind die beiden wichtigsten Selektionsmerkmale die in den Programmen der französischen Forstpflanzenziuchtung Verwendung finden in der Absicht, die Wirksamkeit der Züchtungsarbeit in einem ersten Stadium zu erhöhen. 
Man muss sich dabei jedoch fragen, ob durch die Vernachlässigung anderer, wirtschaftlich wichtiger Merkmale wie z.B. die Rohdichte des Holzes nicht indirekt nachteilige Auswahlen getroffen werden.

Der vorliegende Versuch betrifft 16 Fichtenprovenienzen, vowiegend aus Tief- oder Mittellagen. Die Analysen wurden an vierjährigen Pflanzen durchgeführt, wobei die Variation sowohl zwischen als auch innerhalb der Populationen untersucht wurde.

Die Rohdichtemessungen erfolgten an Stammstücken von $3 \mathrm{~cm}$ Länge, welche oberhalb des Wurzelhalses entnommen wurden. Vier verschieden Rohdichtebestimmungen wurden nach 3 Methoden durchgeführt :

- Viertägige Sättigung im Vakuum (Bestimmung $\mathrm{n}^{\circ} 1$ ).

- Quecksilber-Volumeter (Bestimmung n"2).

- Sättigung durch «Abkochen» während 48 Stunden (Bestimmung $n^{\circ} 3$ und $n^{\circ} 4$ ).

Die verschiedenen Fichtenprovenienzen unterscheiden sich bezüglich der Rohdichte des gebildeten Holzes, wobei die Herkünfte aus dem baltischen Raum am Ende der Reihung zu stehen kommen.

Bezüglich der Mittelwerte der Provenienzen können die verschiedenen festgestellten Merkmalsbeziehungen wie folgt zusammengefasst werden :

Zwischen der Rohdichte des Holzes und den Dimensionsmerkmalen (Höhe und Durchmesser sowie dem Merkmal «Spättreiben» scheint ein negative Beziehung zu bestehen, während die Merkmale «Häufigkeit der Johannistriebbildung» und «Mittlere Andauet des Höhenwachstums » eine positive Korrelation ergeben. Tatsächlich handelt es sich jedoch nur um Tendenzen da nur die Korrelation Rohdichte: Spättreiben signifikant ist. Trägt man der geringen Anzahl der untersuchten Provenienzen Rechnung so kann diesen Ergebnissen kein allgemein giltiger Charakter beigemessen werden.

Bei den Untersuchungen auf individueller Ebene innerhalb der Provenienzen ergaben sich annähernd die gleichen Beziehungen als bei den Mittelwerten zwischen den Provenienzen.

Als Schlussfolgerung muss festgehalten werden, dass die Auswahl von Populationen oder Einzelbäumen nach den Merkmalen «Wuchskraft 》 (vigueur) und «Spättreiben 》 bei Fichte auf Tief- und Mittellagen, die Gefahr einer Verringerung der Rohdichte des Holzes mit sich bringen kann, wenn nicht gewisse Vorsichtsmassregeln getroffen werden.

\section{REFERENCES BIBLIOGRAPHIQUES}

LaCaZe J.-F., 1969. Etude de la variabilité infraspécifique de l'épicéa (Picea abies Karst.). Provenances françaises et polonaises. Résultats au stade juvénile. Ann. Sci. forest., 26, (3), 303-354.

LARSON Ph. R., 1960. Physiological consideration of the spring-wood summervood transition in Red Pine. Forest. Science, 6 (2).

LaRson Ph. R., 1963. The indirect effect of drought on tracheid diameter in Red Pine. Forest Science, 9, (1).

Polge H., 1968. Tests précoces de la qualité du bois sur 25 provenances d'Abies grandis. Ann. Sci. forest., 25, (1), 3-23. 